\title{
Response: Galactose treatment in focal segmental glomerulosclerosis
}

\author{
Asha Moudgil • Kristen Sgambat
}

Received: 6 November 2013 /Revised: 6 November 2013 / Accepted: 8 November 2013 / Published online: 15 January 2014

(C) IPNA 2014

Dear Editors,

We would like to thank the authors of this "letter to the editor" for their interest in our study and for sharing their perspective [1]. We are in agreement with many of the points noted in the letter and have discussed them in our manuscript. The in vitro studies of Savin et al. [1], as well as the case reports by Kopac [2] and De Smet [3], were the catalyst for undertaking this investigation, and are reviewed in our manuscript [4]. The authors state that the lack of response to galactose in our study may be due to preexisting permanent irreversible glomerular damage; we agree with this, and have delineated this as a possible explanation in our discussion section. We have also discussed in our manuscript the possibility that a higher dose of galactose or longer duration of therapy could have yielded different results. We fully agree that there may be subgroups of patients that would benefit from galactose; we therefore proposed further investigation of galactose as a preemptive therapy for prevention of post-transplant focal segmental glomerulosclerosis (FSGS) recurrence, as our permeability activity results suggest a greater chance of response in this subgroup. This does not exclude the possibility that other subgroups of patients may respond to galactose, however at the present time there is no evidence to support this, based on our data. As our study is the first published prospective trial of galactose therapy in steroid-resistant nephrotic syndrome (SRNS), our conclusion stating that galactose is presently of uncertain clinical benefit is based on our results. While galactose is safe and non-toxic, it is also a very expensive therapy and merits further proof of its benefit. We look forward to learning about any new data, including the authors' unpublished observations, that will yield further insight as to if, when, how, and in which subgroup of children with SRNS, this novel therapy can be utilized to achieve partial or complete remission of disease.

\section{References}

1. Trachtman H, Savin V (2013) Galactose treatment in FSGS. Pediatr Nephrol doi: 10.1007/s00467-013-2700-8

2. Kopac M, Meglic A, Rus RR (2011) Partial remission of resistant nephrotic syndrome after oral galactose therapy. Ther Apher Dial 15:269-272

3. De Smet E, Rioux JP, Ammann H, Deziel C, Querin S (2009) FSGS permeability factor-associated nephrotic syndrome: remission after oral galactose therapy. Nephrol Dial transplant 24:2938-2940

4. Sgambat K, Banks M, Moudgil A (2013) Effect of galactose on glomerular permeability in steroid-resistant nephrotic syndrome. Pediatr Nephrol 28:2131-2135

A. Moudgil $(\bowtie) \cdot K$. Sgambat

Children's National Medical Center, Washington, DC, USA

e-mail: amoudgil@childrensnational.org 